\title{
Study of the blood-aqueous barrier in choroidal melanoma
}

\author{
Alain P Castella, Ludmila Bercher, Leonidas Zografos, Emmanuel Egger, Carl P Herbort
}

Department of Ophthalmology, Hôpital Jules Gonin,

Lausanne, Lausanne,

Switzerland

A P Castella

L Bercher

L Zografos

C P Herbort

Paul Scherrer Institut, Swiss National

Institute in Nuclear

Research, Villigen,

Switzerland

E Egger

'La Source' Eye

Center, Lausanne, Switzerland

C P Herbort

Correspondence to: Carl P Herbort, MD, 'La Source' Eye Center, 2 Avenue des Bergières, CH-1004 Lausanne, Switzerland.

Accepted for publication 9 December 1994 University of

\begin{abstract}
Aims-Aqueous flare was used to determine the frequency and amount of bloodaqueous barrier breakdown and correlate it with tumour variables.

Methods-Aqueous flare was analysed prospectively by laser flare photometry in 139 consecutive patients seen in the oncology unit for choroidal melanoma. Both eyes of patients were examined with a laser flare cell meter in a standard fashion.
\end{abstract}

Results-Mean flare difference between healthy and tumour eyes was 3.01 (SD 2.5) photons per millisecond (ph/ms) in 32 cases of small melanomas $(p<0.0001)$, $10.74(13.9) \mathrm{ph} / \mathrm{ms}$ in 92 cases of medium and large melanomas $(p<0.0001)$, and $19.23(11.8) \mathrm{ph} / \mathrm{ms}$ in 15 cases of very large melanomas $(p<0.0001)$. This mean differential flare was significantly higher in medium and large than in small melanomas $(p<0.002)$ and in very large melanomas than in medium and large melanomas $(p<0.028)$. A difference of $\geqslant 7 \mathbf{p h} / \mathbf{m s}$ between affected and healthy eyes was noted in 70 of 139 melanomas $(50 \cdot 4 \%)$. It was found in $3 / 32$ small melanomas (9.4\%), in 53/92 medium and large melanomas $(57 \cdot 6 \%)$, and in $14 / 15$ very large melanomas $(93 \cdot 3 \%)$.

Conclusion-Multiple linear regression analysis showed that flare was most strongly correlated with tumour volume $(r=0.43 ; p<0.0001)$ and tumour height $(r=0.41 ; \mathbf{p}<0.0008)$.

(Brf Ophthalmol 1995; 79: 354-357)

The growth of choroidal melanomas at some point affects adjacent ocular structures such as Bruch's membrane, the retinal pigment epithelium, the sensory retina, the vitreous, the sclera, and the optic nerve. The signs and symptoms of choroidal melanomas are mainly determined by the alteration of these structures. ${ }^{1}$ Data on blood-aqueous barrier in choroidal melanoma are scarce because aqueous flare is usually subclinical and cannot be evaluated precisely with the slit-lamp. ${ }^{2}$

Table 1 Patient and tumour characteristics

\begin{tabular}{llll}
\hline & $S M$ & $M L M$ & $V L M$ \\
\hline Number of patients & 32 & 92 & 15 \\
Age (years (SD)) & $58(2 \cdot 4)$ & $55(1 \cdot 6)$ & $59(3 \cdot 5)$ \\
Sex (MMF) & $14 / 18$ & $48 / 44$ & $7 / 8$ \\
Side (R/L) & $15 / 17$ & $50 / 42$ & $6 / 9$ \\
Mean diameter & $2 \cdot 69(0 \cdot 22)$ & $17 \cdot 2(4 \cdot 3)$ & $10 \cdot 45(0.53)$ \\
Mean height (SD) & $5 \cdot 6(2 \cdot 7)$ & $1045)$ \\
\hline
\end{tabular}

SM=small melanomas; $M L M=$ medium and large melanomas; VLM=very large melanomas.
Laser flare photometry (Kowa laser flare cell meter FC-1000, Kowa Electronics and Optics, Tokyo, Japan) represents an enormous gain of sensitivity over classic slit-lamp evaluation of aqueous flare enabling an objective, quantitative, and non-invasive evaluation that is sufficiently accurate to measure precisely subclinical flare. ${ }^{3-5}$ We used laser flare photometry to evaluate anterior chamber (aqueous) flare in patients with choroidal melanomas in order to determine the frequency and amount of blood-aqueous barrier breakdown and correlate it with different tumour variables.

\section{Patients and methods}

A total of 155 consecutive patients referred to the oncology unit during a period of 10 months with the diagnosis of choroidal melanoma were examined by laser flare photometry. The diagnosis of choroidal melanoma was made on the basis of standard clinical examination and paraclinical investigations in all patients. Depending on their size choroidal melanomas were subdivided into three groups, the demographics of which are detailed in Table 1:

(1) small melanomas (SM) with a diameter of less than $10 \mathrm{~mm}$ and a height of less than $3 \mathrm{~mm}$;

(2) medium and large melanomas (MLM) with a diameter of $10-20 \mathrm{~mm}$ and a height of 3-10 mm;

(3) very large melanomas (VLM) with a diameter $>20 \mathrm{~mm}$ and/or a height $>10 \mathrm{~mm}$.

Fourteen eyes with choroidal melanoma complicated by a neovascular glaucoma, an invasion of the optic nerve, a total retinal detachment, or a diffuse transcleral exteriorisation were excluded from the study. Two patients with a previous contralateral enucleation who had a melanoma in the remaining eye were also excluded. A total of 139 patients were included in the study. Laser flare photometry was performed in both eyes 30 minutes after instillation of dilating drops by the same technician, who did not know which eye the tumour was in. Measurement is based on the backscattering of light produced in the anterior chamber by a helium-neon laser beam of constant power. Backscattered photons are detected with a photomultiplier and analysed with a computer. ${ }^{3}$ Flare intensity is proportional to the protein content of aqueous humour, itself reflecting blood-aqueous barrier disruption. Moreover, flare intensity (scattered light) is proportional to the size of molecules present in the aqueous. ${ }^{6}$ Anterior chamber proteins are mainly composed of albumin but also of a proportion of proteins of higher molecular weight such as globulins. ${ }^{7}$ As the 
Table 2 Anterior chamber flare in choroidal melanoma

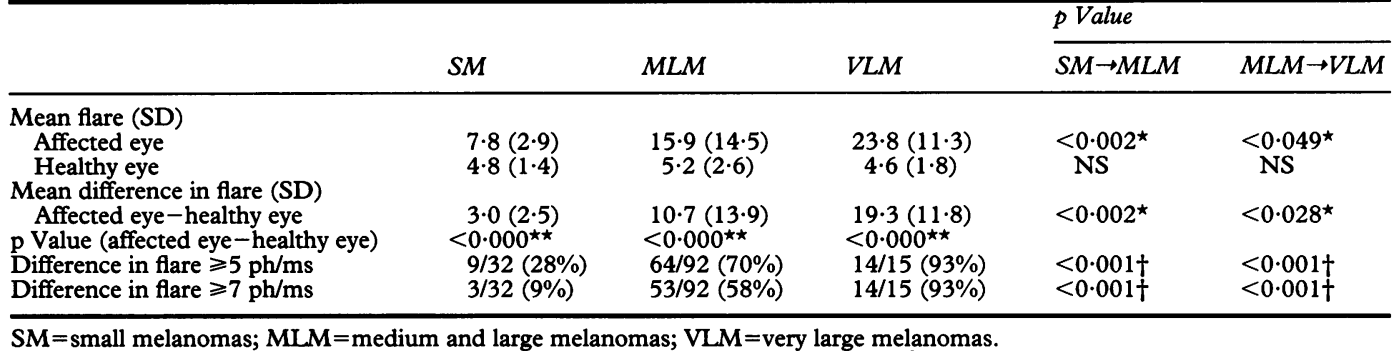

SM=small melanomas; $M L M=$ medium and large melanomas; $V L M=$ very large melanoma

${ }^{\star}=$ Student's unpaired $t$ test (Bonferroni correction); ${ }^{\star \star}=$ Student's paired $t$ test; $\dagger=\chi^{2}$ test.

exact composition of inflammatory aqueous humour is variable, the standard flare units now routinely used are photon counts per millisecond ( $\mathrm{ph} / \mathrm{ms})$, rather than traditionally reported bovine albumin equivalent values expressed in milligrams per millilitre. ${ }^{8}$ Presently a flare increase of $5-7 \mathrm{ph} / \mathrm{ms}$ is empirically considered to be clinically relevant. ${ }^{4}$ In the particle measurement mode, the laser flare cell meter counts cells or other particles in a volume of $0.075 \mathrm{~mm}^{3}$ and the units used are number of cells per $0.075 \mathrm{~mm}^{3}$. The instrument is programmed to count only particles of a size between 9-12 $\mu \mathrm{m}$, ideally counting only inflammatory cells. ${ }^{3}$

Flare difference between the affected (tumour) eye and the healthy eye was statistically analysed using Student's paired $t$ test. Flare values (absolute mean flare and mean differential flare between affected (tumour) and healthy eye) between groups were statistically analysed, using Student's unpaired $t$ test, applying Bonferroni's correction when multiple comparisons were made. The proportion of clinically relevant flare increases $(\geqslant 5 \mathrm{ph} / \mathrm{ms}$ and $\geqslant 7 \mathrm{ph} / \mathrm{ms}$ ) between groups was analysed using the $\chi^{2}$ test.

All medium and large melanomas $(n=92)$ were treated by proton beam irradiation. For this more homogeneous group computerised tumour variables were available and were correlated with the flare value. For this purpose the 92 patients of this group were subdivided into three subgroups according to flare value: flare $<10 \mathrm{ph} / \mathrm{ms}$, flare from 10 to $20 \mathrm{ph} / \mathrm{ms}$, and flare $>20 \mathrm{ph} / \mathrm{ms}$.

The tumour variables studied were: (1) tumour height $(\mathrm{H}=\mathrm{mm})$, determined by $B$ scan real time ultrasonography performed by two different examiners; (2) the largest tumour diameter $(\mathrm{LTD}=\mathrm{mm})$ determined by a computerised mapping of the tumour serving for the proton beam treatment plan; (3) the tumour volume $\left(\mathrm{V}=\mathrm{mm}^{3}\right)$, calculated by a mathematical formula using the largest and smallest tumour diameter and height; (4) the location of the anterior margin of the tumour with reference to the equator of the globe determined with the same computerised mapping and expressed in negative $(-) \mathrm{mm}$ when posterior to the equator and in positive ( + ) $\mathrm{mm}$ when anterior to the equator; (5) the ciliary body invasion determined preoperatively by transillumination and by indirect binocular ophthalmoscopy (percentage of + cases; (6) Bruch's membrane rupture, estimated clinically by indirect ophthalmoscopy, and/or by fluorescein angiography, and/or by ultrasonography (percentage of + cases); (7) retinal detachment estimated clinically by indirect binocular ophthalmoscopy (0-1-2-3 quadrants); and (8) the delay from diagnosis to flare determination (months). Flare was correlated with these eight tumour variables using a multiple linear regression model.

\section{Results}

In all three studied groups (small, medium and large, and very large tumours) the absolute flare values were significantly higher in the tumorous eye than in the healthy eye (Table 2). There was also a significant linear progression from the small to the mediumlarge group and from the medium-large to the very large group both for mean absolute flare and mean differential flare (Table 2). The proportion of cases with a flare difference between paired eyes of $5 \mathrm{ph} / \mathrm{ms}$ or more and $7 \mathrm{ph} / \mathrm{ms}$ or more also significantly increased from one group to the other (Table 2). No significant difference of anterior chamber cells was found between paired eyes in any of the three groups.

For the group of medium-large melanomas treated by proton beam irradiation, the eight clinical and computer generated variables were compared in the three flare subgroups (flare $<10$ 10-20; flare $>20$ ) using paired Student's

Table 3 Correlation of anterior chamber flare and tumour variables (SD) in choroidal melanoma

\begin{tabular}{|c|c|c|c|c|c|}
\hline & $1(n=32)$ & $2(n=40)$ & $3(n=20)$ & $p$ Value ${ }^{*}$ & \\
\hline & $\begin{array}{l}\text { Flare }(\mathrm{ph} / \mathrm{ms}) \\
<10\end{array}$ & $10-20$ & $>20$ & $1 \rightarrow 2$ & $2 \rightarrow 3$ \\
\hline $\begin{array}{l}\text { Volume (mm }) \\
\text { LTD (mm) } \\
\text { Height (mm) } \\
\text { Anterior margin (mm) } \\
\text { Ciliary body invasion (\%) } \\
\text { Bruch's membrane rupture (\%) } \\
\text { Retinal detachment (quadrants) } \\
\text { Diagnosis delay (months) }\end{array}$ & $\begin{array}{l}436(368) \\
14 \cdot 6(4 \cdot 1) \\
3 \cdot 9(1 \cdot 9) \\
-1 \cdot 6(6 \cdot 9) \\
12 \\
22 \\
0 \cdot 74(0.68) \\
6 \cdot 8(11 \cdot 8)\end{array}$ & $\begin{array}{l}896(537) \\
18 \cdot 5(3 \cdot 9) \\
5.9(2 \cdot 3) \\
3 \cdot 1(6 \cdot 3) \\
25 \\
62 \\
1.21(0.75) \\
4.9(6 \cdot 1)\end{array}$ & $\begin{array}{l}1318(629) \\
19 \cdot 1(3 \cdot 2) \\
7 \cdot 8(2 \cdot 8) \\
6 \cdot 1(5 \cdot 8) \\
45 \\
60 \\
1 \cdot 27(0 \cdot 82) \\
5 \cdot 4(7 \cdot 6)\end{array}$ & $\begin{array}{l}0 \cdot 0001 \\
0 \cdot 0003 \\
0 \cdot 0005 \\
0 \cdot 0026 \\
\text { NS } \\
0 \cdot 0002 \\
0 \cdot 0097 \\
\text { NS }\end{array}$ & $\begin{array}{l}0 \cdot 025 \\
\text { NS } \\
0 \cdot 021 \\
0 \cdot 098 \\
\text { NS } \\
\text { NS } \\
\text { NS } \\
\text { NS }\end{array}$ \\
\hline
\end{tabular}

LTD $=$ largest tumour diameter. ${ }^{\star}$ Student's unpaired $t$ test (Bonferroni correction). 


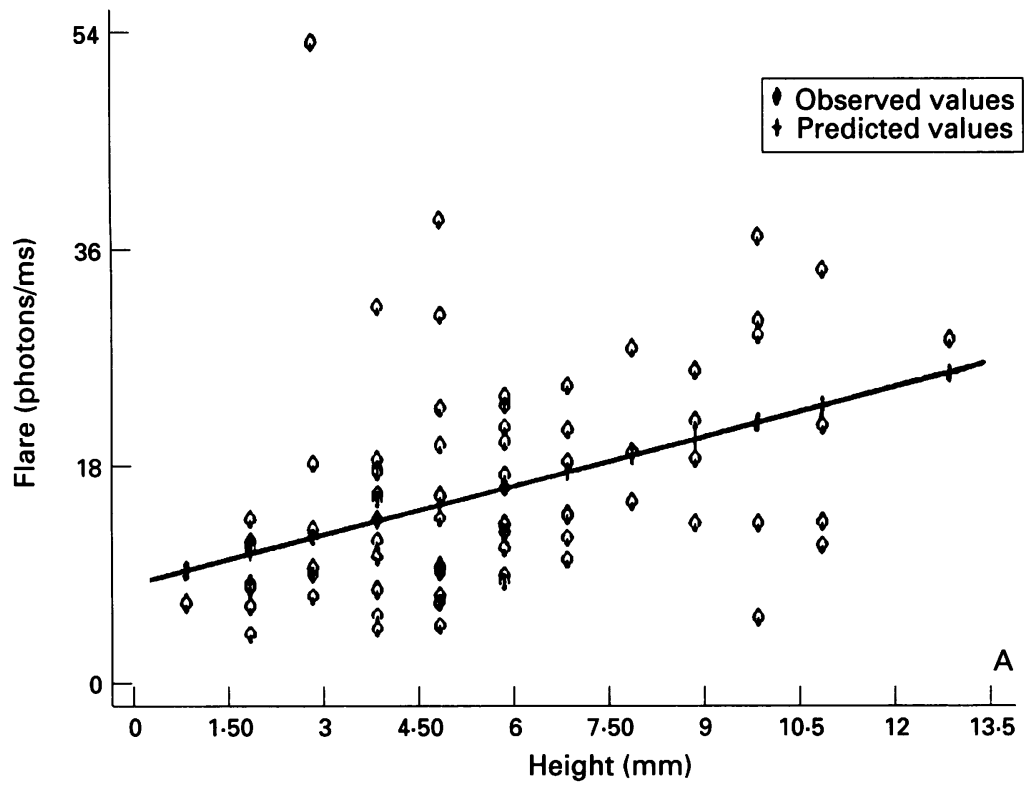

Flare $=6.80+1.3513 * \mathrm{H}$

Regression coefficient: 0.41

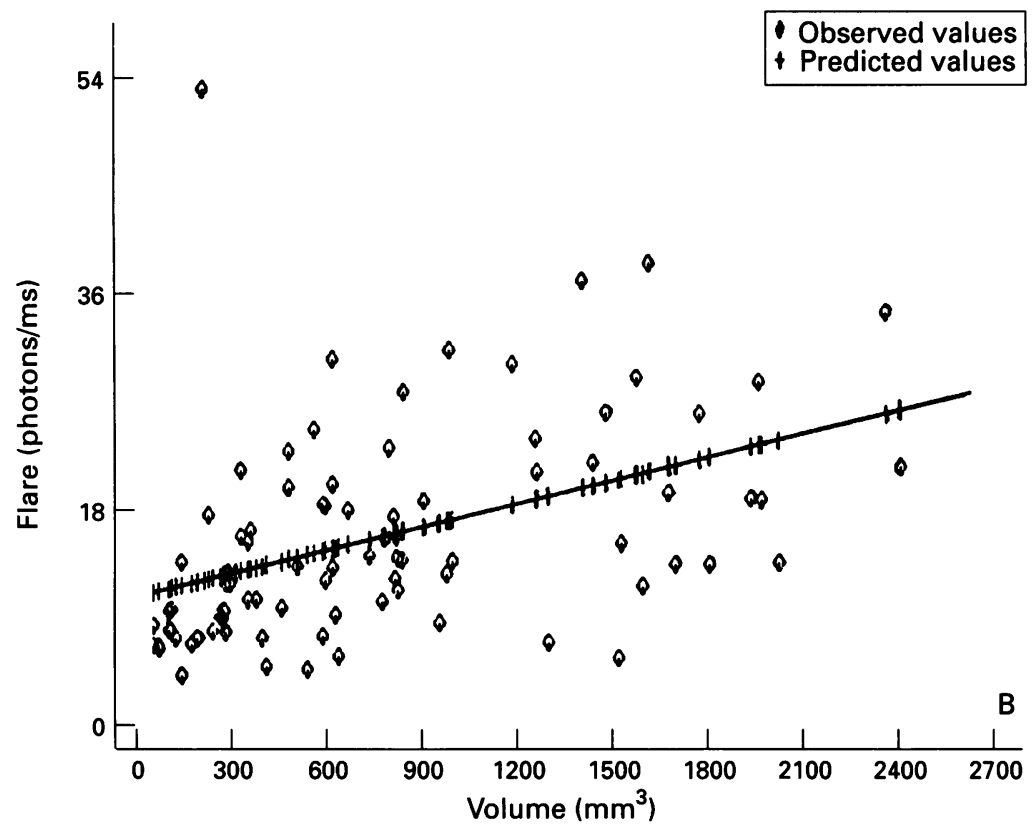

Flare $=9.20+0.0063 * \mathrm{~V}$

Regression coefficient: 0.43

Figure 1 Regression line of flare values (photons/ms) in correlation with tumour height $(\mathrm{mm})(A)$ and tumour volume $\left(\mathrm{mm}^{3}\right)(B)$.

$t$ test (Table 3). There was a significant, linear progression from subgroup 1 to subgroup 2 and from subgroup 2 to subgroup 3 only for tumour volume and tumour height, indicating that flare increase seemed to be related to tumour volume and height (Table 3 ). This was confirmed when these variables were analysed by multiple regression analysis. Flare increase showed a significant correlation with tumour volume $(r=0.43), \mathrm{p}<0.0001 ;$ Fig $1 \mathrm{~A})$ and tumour height $(r=0.41, \mathrm{p}<0.0008$; (Fig 1B), but not to largest tumour diameter $(r=0.27$, $\mathrm{p}=\mathrm{NS}$ ), location of the anterior margin of the tumour $(r=0.32, \mathrm{p}=\mathrm{NS})$, ciliary body invasion $(r=0 \cdot 23, \mathrm{p}=\mathrm{NS})$, associated retinal detachment $(r=0.22, \mathrm{p}=\mathrm{NS})$, Bruch's membrane rupture $(r=0 \cdot 26, p=N S)$, or to delay from diagnosis to flare determination $(r=0 \cdot 05, \mathrm{p}=\mathrm{NS})$.

\section{Discussion}

Breakdown of the blood-aqueous barrier resulting in an influx of proteins (flare) can be caused by inflammation or by any other as yet unexplained non-inflammatory insult to the blood-aqueous barrier such as in diabetes. ${ }^{10}$ Blood-aqueous barrier disruption associated with choroidal melanomas was only rarely reported even in large series. In a group of 450 melanomas examined with the slit-lamp, Fraser et al reported an elevated flare in the anterior chamber in $4.9 \%$ of the cases. ${ }^{2}$ Using laser flare photometry we found that anterior chamber flare was elevated much more often in choroidal melanoma than previously suspected by slit-lamp examination. Flare was significantly higher in the tumorous eye when compared with the healthy fellow eye in groups of melanomas of all sizes. An empirically determined increase of flare of $5-7 \mathrm{ph} / \mathrm{ms}$ or more is presently considered clinically relevant. ${ }^{49}$ An increase of $\geqslant 5 \mathrm{ph} / \mathrm{ms}$ occurred in $62.6 \%$ and an increase of $\geqslant 7 \mathrm{ph} / \mathrm{ms}$ occurred in $50.4 \%$ of all cases and reached $93.3 \%$ in the group of very large melanomas, indicating that the tumour was causing a distinct breakdown of the blood-aqueous barrier in a significant number of cases. The amount of flare was linearly correlated with the size of the melanoma, being highest in the group of very large melanomas.

Multiple linear regression coefficients showed that the amount of flare was related to the size of the tumour, as flare value was clearly correlated both with tumour volume and height. The location of the tumour and whether it invaded the ciliary body or not, Bruch's membrane rupture, the associated retinal detachment, and the delay from diagnosis to flare determination did not influence the amount of flare, indicating that, except for size (volume and height), no other clinical variable influenced the amount of blood-aqueous barrier breakdown. The reason why melanomas cause blood-aqueous breakdown is not known and can only be hypothesised. Recent experimental studies demonstrated the proinflammatory properties of melanin or of a melanin associated protein. In a mouse uveitis model where presensitised animals were challenged in the anterior chamber with horse serum or conalbumin, melanin was shown to increase inflammation. ${ }^{11}$ Binding of melanin to serum components such as antibodies was thought to be a possible explanation for its proinflammatory activity. ${ }^{11}$ Broekhuyse et al and other groups recently reported that a protein fraction extracted from the retinal pigment epithelium and from the choroid acted as an autoantigen and produced an autoimmune anterior uveitis in rats, called experimental autoimmune anterior uveitis (EAAU). ${ }^{12} 13$ EAAU could also be elicited with an extract from the iris and ciliary body. ${ }^{14}$ If a melanin associated protein is responsible for bloodaqueous breakdown, it seems logical that tumours of great size containing a larger amount of protein associated with melanogenesis would have an increased tendency to produce a higher degree of blood-aqueous 
barrier breakdown. Other factors that possibly might influence blood-aqueous barrier breakdown are alterations of retinal vascular permeability overlying melanomas, lymphocytic infiltration, and amount of necrosis of the tumour. ${ }^{1516}$

The most likely hypothesis, however, is that choroidal melanomas cause blood-aqueous barrier disruption in a similar fashion to diabetes. It was shown by laser flare photometry that the amount of diabetes associated blood-aqueous barrier disruption was directly related to the degree of diabetic retinopathy. ${ }^{1017}$ These changes in blood-ocular barrier permeability are thought to be caused by diffusible angiogenic factors generated by hypoxic tissue. One of these factors, vasculotropin/vascular endothelial growth factor (VAS/VEGF) that has been recently purified controls both angiogenesis and vascular permeability and was found to be significantly elevated in vitreous of patients with proliferative diabetic retinopathy. ${ }^{1819}$ Tumours also produce similar 'tumour angiogenic factors' causing blood vessels from adjacent normal tissue to grow towards and into the tumour providing the tumour with oxygen and other nutrients. As in diabetic retinopathy where flare is proportional to the extent of ischaemic areas, flare was directly related to the extent of the tumour (melanoma size). Another similarity to proliferative diabetic retinopathy is the neovascular glaucoma that can develop after irradiation of large choroidal melanomas despite limited irradiation of the adjacent retina obtained with the proton beam. This could be the result of massive release of diffusible angiogenic factors from hypoxic tumour tissue and/or radiation retinopathy as in proliferative diabetic retinopathy. ${ }^{2021}$

Finally, it is worth stressing that laser flare photometry should not be considered as a sensitive diagnostic help in the examination of choroidal melanomas as suggested earlier in a study on 11 melanomas. ${ }^{22}$ Taking the lower value $(5 \mathrm{ph} / \mathrm{ms})$ of what is considered a clinically relevant flare rise, more than one third of melanomas $(37 \cdot 4 \%)$ had an increase of less than $5 \mathrm{ph} / \mathrm{ms}$ and in the group of small melanomas this proportion was as high as $72 \%$.
1 Shields JA. Posterior uveal melanomas: clinical and pathologic features. In: Shields JA, ed. Diagnosis and management of intraocular tumors. St Louis: Mosby, 1983: managem

2 Fraser DJ, Font RL. Ocular inflammation and hemorrhage as initial manifestations of uveal malignant melanoma. Arch Ophthalmol 1979; 97: 1311-4.

3 Sawa M, Tsurimaki Y, Tsuru T, Shimizu H. New quantitative method to determine protein concentration and cell number in aqueous in vivo. $f_{p} n \mathcal{F}$ Ophthalmol 1988; 32 132-42.

4 Altamirano O, Mermoud A, Pittet N, van Melle G, Herbort $\mathrm{CP}$. Aqueous humor analysis after Nd:YAG laser capsulotomy with the laser flare-cell meter. $¥$ Cataract Refract lotomy with the laser
Surg 1992; 18: 554-8.

5 Guex-Crosier Y, Pittet N, Herbort CP. Evaluation of laser flare-cell photometry in the appraisal and management of intraocular inflammation in uveitis. Ophthalmology 1994 101: 728-35.

6 Shah SM, Spalton DJ, Taylor JC. Correlations between laser flare measurements and anterior chamber protein concentrations. Invest Ophthalmol Vis Sci 1992; 33: 2878-84.

7 Zirn M. Proteins in aqueous humor. Adv Ophthalmol 1980; 40: $100-72$.

8 Sawa $M$. Clinical application of laser flare-cell meter. $f_{p n} \mathcal{f}$ Ophthalmol 1990; 34: 346-63.

9 Herbort CP, Mermoud A, Schnyder C, Pittet N. Antiinflammatory effect of diclofenac drops after argon lase trabeculoplasty. Arch Ophthalmol 1993; 111: 481-3.

10 Moriarty AP, Spalton DJ, Moriarty BJ, Shilling JS, Ffytche TJ, Bulsara M. Studies of blood-aqueous barrier in diabetes mellitus. Am $\mathcal{f}$ Ophthalmol 1994; 118: 768-71.

11 Kaya M, Edward DP, Tessler H, Hendricks RL Augmentation of intraocular inflammation by melanin. Invest Ophthalmol Vis Sci 1992; 33: 522-31.

12 Broekhuyse RM, Kuhlmann ED, Winkens HJ, Van Vugt AH. Experimental autoimmune anterior uveitis (EAAU), a new form of experimental uveitis. Induction by a a new form of experimental uveitis. Induction by a retinal pigment epithelium. Exp Eye Res 1991; 52 465-74.

13 Chan CC, Hikita N, Dastgheib K, Whitcup SM, Gery I, Nussenblatt RB. Immunopathology of experimenta autoimmune anterior uveitis (EAAU). Invest Ophthalmol Vis Sci 1993; 34: 999.

14 Kaplan AD, Bora NS, Gobleman CL, Kaplan HJ. Experimental allergic iritis a new model of autoimmune uveitis. Invest Ophthalmol Vis Sci 1993; 34: 1480.

15 Cantrill HL, Cameron DJ, Ramsay RC, Knobloch WH. Retinal vascular changes in malignant melanoma of the Retinal vascular changes in malignant mela
choroid. Am $₹$ Ophthalmol 1984; 97: 41 1-8.

16 Rahi AHS, Agrawal PK. Prognostic parameters in choroidal melanoma. Trans Ophthalmol Soc UK 1977; 97 368-72.

17 Oshika T, Kato S, Funatsu H. Quantitative assessment of aqueous flare intensity in diabetes. Graefes Arch Clin Exp Ophthalmol 1989; 227: 518-20.

18 Favard C, Moukadiri H, Dorey C, Praloran V, Plouet J. Purification and biological properties of vasculotropin, a new angiogenic cytokine. Biol Cell 1991; 73: 1-6.

19 Favard C, Jonca F, Ruchoux MM, Plouet J. Angiogenic potency of vasculotropin/VEGF is related to posttraducpotency of vasculotropin/VEGF is related to posttraduc1499 .

20 Kincaid MC, Folberg R, Torczynski E, Zakov ZN, Shore $\mathrm{JW}$, Liu SJ, et al. Complications after proton beam therapy for uveal malignant melanoma. Ophthalmolog 1988; 95: 982-91.

21 McFaul PA, Morgan G. Histopathological changes in malignant melanomas of the choroid after cobalt therapy. Br f Ophthalmol 1977; 61: 221-8.

22 Küchle M, Nguyen NX, Naumann GOH. Aqueous flare in eyes with choroidal malignant melanoma. $\mathrm{Am} f$ Ophthalmol 1992; 113: 207-8. 